\title{
A Survey Among Italian Pregnant Women and New-mothers During the COVID-19 Pandemic Lockdown.
}

\section{Viviana STAMPINI ( $\sim$ viviana.stampini@med.uniupo.it)}

Universita degli Studi del Piemonte Orientale Amedeo Avogadro Dipartimento di Medicina Traslazionale https://orcid.org/0000-00021183-0603

\section{Alice MONZANI}

Universita degli Studi del Piemonte Orientale Amedeo Avogadro

\section{Silvia CARISTIA}

Universita degli Studi del Piemonte Orientale Amedeo Avogadro

\section{Gianluigi FERRANTE}

CPO-Piemonte

\section{Martina GERBINO}

Universita degli Studi del Piemonte Orientale Amedeo Avogadro

Roberta AMADORI

Azienda Ospedaliero-Universitaria Maggiore della Carita

Ivana RABBONE

Universita degli Studi del Piemonte Orientale Amedeo Avogadro

\section{Daniela SURICO}

Universita degli Studi del Piemonte Orientale Amedeo Avogadro

\section{Research article}

Keywords: COVID-19, Lockdown, Pregnancy, New-mothers, Breastfeeding, Healthy eating, Physical exercise

Posted Date: August 10th, 2020

DOI: https://doi.org/10.21203/rs.3.rs-50602/v1

License: (1) (i) This work is licensed under a Creative Commons Attribution 4.0 International License. Read Full License 


\section{Abstract}

In response to the COVID-19 pandemics, drastic measures for social distancing have been introduced also in Italy. The purpose of this study was to describe some aspects of lifestyle, access to health services, and mental wellness of Italian pregnant women and newmothers during the lockdown.

We carried out a web-based survey to assess how pregnant women and new-mothers were coping with the lockdown. Expected outcomes were categorized in different analysis domains: psychological well-being and support, physical exercise, dietary habits, access to care, delivery and obstetric care, neonatal care and breastfeeding.

We included 742 respondents (response rate $86.2 \%), 603$ were pregnant $(81.3 \%)$ and 139 (18.7\%) had delivered during lockdown. We found a high score for anxiety and depression in $62.7 \%$ of pregnant women and $61.9 \%$ of new-mothers. During the lockdown, $61.9 \%$ of pregnant women reduced their physical exercise, and 79.8\% reported to eat in a healthier way. $94.2 \%$ of new-mothers reported to have breastfed their babies during hospital staying. Regarding the impact of restrictive measures on breastfeeding, no impact was reported by $56.1 \%$ of new-mothers, a negative impact by $36.7 \%$.

The higher prevalence of anxiety and depressive symptoms in pregnant women and new-mothers should be a public health issue. Clinicians might also consider to recommend and encourage "home" physical exercise. On the other hand, most women improved their approach towards healthy eating during the lockdown and a very high breastfeeding rate was reported soon after birth: these data are an interesting starting point to develop new strategies for public health.

\section{Introduction}

Since severe acute respiratory syndrome SARS-CoV-2 started to spread across several countries, the World Health Organization declared that the outbreak was a public health emergency of international concern (1).

Based on the Chinese experience (2), starting from March 9th, 2020, drastic measures have been introduced also in Italy: citizens are banned from leaving their home unless for daily needs that cannot be postponed. As a consequence, a sudden and radical change in habits and lifestyles of the whole population, a minimization of socialization and changes in both interpersonal relationships and organization of work occurred.

Hospital activity has radically changed: many Departments were closed in order to create COVID-19 dedicated hospital wards, the rest of the clinical activity was downsized and contacts with patients were reduced to the minimum. Measures such as re-adaptation of care priorities and several restrictions will presumably lead to changes in the health of the population in the coming months or years.

There are still many unanswered questions regarding the effect of lockdown measures on pregnant women (3) (4). Healthcare workers are facing an important challenge in terms of reshaping obstetric care in order to avoid unnecessary exposure to patients, without renouncing the required attention. Although obstetric units have not diminished their working activity, there have been changes in territorial and hospital care. Some activities whose usefulness is established by evidence, have undergone through major downsizing. The pre-birth courses have been officially stopped; some screening tests have been performed much less frequently, due to reduced patient access to the tests, or due to difficulties in providing the services. Family members and partners presence during important moments, such as ultrasound screens and hospitalization, has been reduced for safety reasons.

Most of the pregnant women and new-mothers found themselves unable to leave their home, often with other children to look after, not able to count on any domestic help (5). On the other hand, some women may have welcomed the chance of working from home or in some cases counting on a larger presence of their partner.

It can be assumed that these changes may have an influence on pregnancy, puerperium and newborn management that obstetricians should consider (4). While published studies on the possible effects of COVID-19 disease in pregnant women and infants are increasing (6), there are only few studies (7) (8) (9) dealing with the psychological effect of the pandemic on pregnant women. To the best of our knowledge, there are still no studies in literature about how mothers are coping with the lockdown and highlighting the needs of this population group in that period.

The purpose of this study is to describe some aspects of lifestyle, access to health services and mental wellness of Italian pregnant women and new-mothers during the lockdown. 


\section{Materials And Methods \\ Study Design, setting and participants}

We carried out a cross-sectional survey by using an anonymous online questionnaire. The survey addressed both pregnant women and women who gave birth during the lockdown. As the Italian government recommended to minimize face-to-face interaction, the questionnaire was web based; individuals were offered the opportunity to participate through social media (pages of Facebook and Instagram dedicated to new-mothers and pregnant women) and through newspapers sections for women issues.

Individuals were directed via an electronic link to an online survey platform. Duplicate entries were avoided by asking people to provide their e-mail address at the end of the survey; duplicate entries having the same e-mail address were eliminated before analysis and the first entry was kept. The survey was not displayed a second time once the responder had filled it in, but the link to pass it on to others was available. Therefore, snowball sampling technique, where existing study subjects recruit future subjects among their acquaintances, was also used. The survey period lasted for 4 weeks through the lockdown period, from April 9th, 2020 to May 3rd, 2020.

\section{Ethical considerations}

The survey was preceded by a fact sheet including information on what the research was about, the reason for conducting the research, how the data will be used, how privacy of data will be maintained, information in case the respondents changed their mind during the survey, along with contact details for further information. Afterward, the consent to participate was required to proceed with the survey. The participation in the survey was voluntary and anonymous. Approval was obtained from the local Ethical Committee (Comitato Etico Interaziendale Novara CE 71/20), which conformed to the principles embodied in the Declaration of Helsinki.

\section{Variables and data sources}

Expected outcomes of the baseline analysis concerned different topics that could be influenced by the lockdown measures, and were categorized in different analysis domains, related to pregnancy and puerperium.

About pregnancy the analyzed domains were 1-psychological well-being and support; 2-physical exercise; 3- dietary habits; 4- access to care. About puerperium the analyzed domains were 5- psychological well-being and support; 6- delivery and obstetric care; 7- neonatal care and breastfeeding.

Due to the lack of validated questionnaires about this topic, authors reviewed previous and current surveys (10) on the impacts of outbreaks and included additional questions related to pregnancy and birth (11) (12) (13). To investigate the psychological impact we used the Patient Health Questionnaire for Depression and Anxiety (the PHQ-4)(14). The questionnaire was tested in a sample of voluntary pregnant women and new-mothers, with different characteristics (age, education, parity) who reviewed the questionnaire individually and provided verbal feedback, and it was also submitted to a panel of experts (psychologist, midwife, epidemiologist), for content validity and construct coherence. Completion time was 15 minutes.

The survey consisted of a common part including sociodemographic and psychological questions, addressing both pregnant women and new-mothers, followed by a specific part dedicated either to pregnant women or women who gave birth during the lockdown, each divided into sub-sections of questions, addressing the different analysis domains.

\section{Statistical analysis}

We analyzed frequency distributions of variables separately for pregnant women and new-mothers, calculated means and standard deviations for continue variables. T-test was used to study the differences between categories in the continuous variable (expressed as mean and SD), while for the categorical variables (expressed as number and percentage of the total) the reference test was chi-square (X2). Level of significance was set with $p<0.05$. Poisson regression multivariate models were used to calculate prevalence ratios and relative $95 \%$ confidence intervals. Statistical analysis was performed using Stata Statistical Software: Release 15. StataCorp LLC.

\section{Results}

We received responses from 861 women (Fig. 1). Eight (0.9\%) women did not give their consent to participate, while 111 (12.9\%) were excluded because either not pregnant or not having given birth during the restrictive measures. We included 742 respondents from 107 
different cities in Italy, who had completed the questionnaires (completion rate: 96\%). Overall, 603 respondents were pregnant (81.3\%) and 139 (18.7\%) respondents had delivered in the lockdown period.

\section{Sociodemographic, living, housing and psychological characteristics of pregnant women and new-mothers}

Data are reported in Table 1. The overall response rate for these items was $>99 \%$. 
Table 1

Socio-demogaphic characteristics, housing and living conditions

\begin{tabular}{|c|c|c|c|c|c|c|}
\hline \multirow{3}{*}{$\begin{array}{l}\text { Socio-demogaphic } \\
\text { characteristics }\end{array}$} & \multirow{3}{*}{$\begin{array}{l}\text { Parameter } \\
\text { Age }\end{array}$} & \multirow{2}{*}{$\begin{array}{l}\text { Category } \\
\text { From } 18 \text { to } 34\end{array}$} & \multicolumn{2}{|c|}{$\begin{array}{l}\text { Number }(\mathrm{N}= \\
603) \%\end{array}$} & \multicolumn{2}{|c|}{$\begin{array}{l}\text { Number }(\mathrm{N}= \\
139) \%\end{array}$} \\
\hline & & & 381 & $63.2 \%$ & 82 & $59.0 \%$ \\
\hline & & From 35 to 48 & 222 & $36.8 \%$ & 57 & $41.0 \%$ \\
\hline & \multirow[t]{2}{*}{ Education } & Less than Bachelor & 230 & $38,2 \%$ & 37 & $26.6 \%$ \\
\hline & & More than bachelor & 372 & $61,8 \%$ & 102 & $73.4 \%$ \\
\hline & \multirow[t]{4}{*}{ Area of residence } & North & 486 & $80,6 \%$ & 125 & $89.9 \%$ \\
\hline & & Centre & 65 & $10,8 \%$ & 12 & $8.6 \%$ \\
\hline & & South & 49 & $8,1 \%$ & 2 & $1.4 \%$ \\
\hline & & Abroad & 3 & $0,5 \%$ & & \\
\hline & \multirow[t]{2}{*}{ City or village } & $\begin{array}{l}\text { City or suburbs of a } \\
\text { city }\end{array}$ & 285 & $47,4 \%$ & 60 & $43,2 \%$ \\
\hline & & Village & 316 & $52,6 \%$ & 79 & $56,8 \%$ \\
\hline & \multirow[t]{2}{*}{ Economical resources } & Not much adeguate & 312 & $52,0 \%$ & 72 & $52,6 \%$ \\
\hline & & Very adeguate & 288 & $48,0 \%$ & 65 & $47,4 \%$ \\
\hline & \multirow[t]{2}{*}{ Working conditions } & Workers & 456 & $75,7 \%$ & 108 & $78,3 \%$ \\
\hline & & Not workers & 146 & $24,3 \%$ & 30 & $21,7 \%$ \\
\hline \multirow[t]{8}{*}{ Housing } & \multirow[t]{2}{*}{ House size } & Less than $100 \mathrm{sm}$ & 389 & $64.5 \%$ & 94 & $67,6 \%$ \\
\hline & & More than $100 \mathrm{sm}$ & 468 & $35.5 \%$ & 45 & $32,4 \%$ \\
\hline & \multirow[t]{2}{*}{ Satisfaction with the house } & Not much & 233 & $38.6 \%$ & 54 & $38,8 \%$ \\
\hline & & Satisfied & 370 & $61.4 \%$ & 85 & $61,2 \%$ \\
\hline & \multirow[t]{2}{*}{ Presence of a garden } & Yes & 253 & $42.0 \%$ & 53 & $38,4 \%$ \\
\hline & & No & 350 & $58.0 \%$ & 85 & $61,6 \%$ \\
\hline & \multirow[t]{2}{*}{ Adeguacy of electronic devices } & Not much adeguate & 137 & $22.7 \%$ & 41 & $29,5 \%$ \\
\hline & & Very adeguate & 466 & $77.3 \%$ & 98 & $70,5 \%$ \\
\hline \multirow[t]{11}{*}{ Social-iving } & \multirow[t]{3}{*}{ Presence of partner } & Always at home & 312 & $51.7 \%$ & 70 & $50,4 \%$ \\
\hline & & $\begin{array}{l}\text { At home but still going } \\
\text { to work }\end{array}$ & 277 & $45.9 \%$ & 65 & $46,8 \%$ \\
\hline & & Not co-living & 14 & $2.3 \%$ & 4 & $2,9 \%$ \\
\hline & \multirow[t]{2}{*}{ Other children at home } & No & 408 & $67.7 \%$ & 99 & $71,2 \%$ \\
\hline & & Yes & 195 & $32.3 \%$ & 40 & $28,8 \%$ \\
\hline & \multirow[t]{2}{*}{ Someone else co-living } & No & 559 & $92.7 \%$ & 123 & $88,5 \%$ \\
\hline & & Yes & 44 & $7.3 \%$ & 16 & $11,5 \%$ \\
\hline & \multirow[t]{2}{*}{ Contacts with other people } & No & 379 & $62.8 \%$ & 71 & $51,4 \%$ \\
\hline & & Yes & 224 & $37.2 \%$ & 67 & $48,6 \%$ \\
\hline & \multirow[t]{2}{*}{ Adherence to the restrictions } & From little to average & 111 & $18.4 \%$ & 26 & $18,7 \%$ \\
\hline & & High & 492 & $81.6 \%$ & 113 & $81,3 \%$ \\
\hline Psycological well being & Depression and anxiety score & Normal to mild & 225 & $37.3 \%$ & 53 & $38,1 \%$ \\
\hline
\end{tabular}




\begin{tabular}{|c|c|c|c|c|c|c|}
\hline \multirow[b]{2}{*}{ and support } & \multirow[t]{2}{*}{ Parameter } & \multirow{2}{*}{$\begin{array}{l}\text { Category } \\
\text { Moderate to severe }\end{array}$} & \multicolumn{2}{|c|}{$\begin{array}{l}\text { Number }(\mathrm{N}= \\
603) \%\end{array}$} & \multicolumn{2}{|c|}{$\begin{array}{l}\text { Number }(\mathrm{N}= \\
139) \%\end{array}$} \\
\hline & & & 378 & $62.7 \%$ & 86 & $61,9 \%$ \\
\hline & \multirow{8}{*}{$\begin{array}{l}\text { People supporting (more than one } \\
\text { choice available) }\end{array}$} & Partner & 502 & $83.2 \%$ & 121 & $87,1 \%$ \\
\hline & & Mother & 395 & $65.5 \%$ & 92 & $36,7 \%$ \\
\hline & & Sister/brothers & 233 & $38.6 \%$ & 51 & $61,2 \%$ \\
\hline & & Friends & 287 & $47.6 \%$ & 60 & $43,2 \%$ \\
\hline & & Gynecologyst & 83 & $13.6 \%$ & 10 & $7,2 \%$ \\
\hline & & Midwife & 68 & $13.6 \%$ & 27 & $19,4 \%$ \\
\hline & & $\begin{array}{l}\text { Other women in } \\
\text { pregnnacy }\end{array}$ & 112 & $18.6 \%$ & 42 & $30,2 \%$ \\
\hline & & Internet pages & 40 & $6.6 \%$ & 8 & $5,7 \%$ \\
\hline
\end{tabular}

Pregnant women were aged 18 to 48 years with a mean of $33.1 \pm 4.3,61.8 \%$ of the sample had at least a bachelor's degree, $48.0 \%$ of the sample said money were highly sufficient to their needs. The PHQ-4 score ranged from moderate to severe for $62.7 \%$ of women. $83.5 \%$ of women recognize the partner as the person who is supporting them during the lockdown.

New-mothers were aged 25 to 41 years with a mean of $33.6 \pm 4.0,73.4 \%$ had at least a bachelor's degree, $47.4 \%$ of the sample said money were highly sufficient to their needs. Similar to pregnant women, also for $61.9 \%$ of new-mothers the PHQ- 4 score ranged from moderate to severe and the partner was the most cited as the person supporting them (87.1\%). Most of respondents were living in Northern Italy.

\section{Changes in the life of pregnant women during the lockdown}

Table 2 describes some aspects of pregnant women's life and how the social distancing measures changed their habits (supporting people, dietary habits, physical exercise, and access to care). The overall response rate for these items was $>96 \%$. Many women (79.1\%) declared the greater presence of the partner positively influences the pregnancy. Besides, $74.3 \%$ of them was afraid of delivering alone and $44.3 \%$ said the current situation generates stress about the future. About physical exercise, the reported minutes of weekly physical activity were significantly decreased during quarantine (145.9 minutes $95 \%$ Cl 137.4-154.5 before versus 105.7 minutes $95 \%$ $\mathrm{Cl} 96.8-114.6$ during the lockdown, $\mathrm{p}<0.05)$. Specifically, $61.9 \%$ of women during the lockdown reduced their physical exercise. Concerning dietary habits, according to $79.8 \%$ of women, restrictions gave them the chance to eat healthier. 
Table 2

Changes in the life of pregnant women during the lockdown

\begin{tabular}{|c|c|c|c|c|}
\hline & Parameter & Category & $\begin{array}{l}N= \\
603\end{array}$ & $\%$ \\
\hline \multirow{10}{*}{$\begin{array}{l}\text { Other psycologycal } \\
\text { aspects }\end{array}$} & Influence of partner at home & Positive influence & 447 & $79,1 \%$ \\
\hline & & Negative influence & 11 & $1,9 \%$ \\
\hline & & No influence & 107 & $18,9 \%$ \\
\hline & Influence of children at home & Positive influence & 52 & $26.7 \%$ \\
\hline & & Negative influence & 72 & $36.9 \%$ \\
\hline & & No influence & 71 & $36.4 \%$ \\
\hline & Fear of delivering alone & Low & 149 & $25,7 \%$ \\
\hline & & High & 431 & $74,3 \%$ \\
\hline & Stress about future & Low to average & 336 & $55.7 \%$ \\
\hline & & High & 267 & $44.3 \%$ \\
\hline \multirow[t]{11}{*}{ Physical exercize } & \multirow[t]{2}{*}{ Weekly exercize before the restrictions } & More than 2 hours & 270 & $58,4 \%$ \\
\hline & & Less than 2 hours & 192 & $41,6 \%$ \\
\hline & \multirow[t]{2}{*}{ Weekly exercize after the restrictions } & More than 2 hours & 140 & $30,3 \%$ \\
\hline & & Less than 2 hours & 322 & $69,7 \%$ \\
\hline & \multirow[t]{3}{*}{ Changes pre-post lock-down of weekly minutes } & As before & 88 & $17.4 \%$ \\
\hline & & More than before & 105 & $20.7 \%$ \\
\hline & & Less than before & 313 & $61.9 \%$ \\
\hline & \multirow[t]{2}{*}{ Restrictions gave you the chance to practice more } & Yes & 87 & $14,4 \%$ \\
\hline & & No & 516 & $85,6 \%$ \\
\hline & \multirow[t]{2}{*}{ Not walking outside: influence on your wellenss } & Low & 487 & $80,9 \%$ \\
\hline & & High & 115 & $19,1 \%$ \\
\hline \multirow[t]{2}{*}{ Dietary habits } & \multirow{2}{*}{$\begin{array}{l}\text { Restrictions gave you the chance to eat more } \\
\text { healthy }\end{array}$} & Yes & 481 & $79,8 \%$ \\
\hline & & No & 122 & $20,2 \%$ \\
\hline \multirow[t]{11}{*}{ Access to care } & \multirow[t]{3}{*}{ Participiation to online pre birth course } & Currently participating & 151 & $25,1 \%$ \\
\hline & & About to start & 10 & $1,7 \%$ \\
\hline & & Not participating & 440 & $73,2 \%$ \\
\hline & \multirow[t]{2}{*}{ Access to emergency room } & Give up to go to ER & 72 & $11,9 \%$ \\
\hline & & No evenience & 531 & $88,1 \%$ \\
\hline & \multirow[t]{4}{*}{ How did you solve the problem $(\mathrm{N}=72)$} & $\begin{array}{l}\text { Phone call with } \\
\text { Gynecologist }\end{array}$ & 34 & $47,2 \%$ \\
\hline & & Phone call with midwife & 17 & $23,6 \%$ \\
\hline & & $\begin{array}{l}\text { Visit to private } \\
\text { Gynecologist }\end{array}$ & 14 & $19,4 \%$ \\
\hline & & Others & 7 & $9,7 \%$ \\
\hline & \multirow[t]{2}{*}{ Have you skipped any planned visit? } & Yes & 159 & $26,4 \%$ \\
\hline & & No & 444 & $73,6 \%$ \\
\hline
\end{tabular}




\begin{tabular}{|llrr|}
\hline Parameter & Category & $\begin{array}{c}\text { N= } \\
603\end{array}$ \\
\hline Have you skipped any planned exam or vaccine? & Yes & 112 & $18,7 \%$ \\
\cline { 2 - 4 } & No & 486 & $81,3 \%$ \\
\cline { 2 - 4 } & & & 403 \\
\hline
\end{tabular}

Regarding the access to health-care services, only $25.1 \%$ of women in our sample was attending an online pre-birth course and $11.9 \%$ of them avoided to go to an ob-gyn emergency room because of the fear of contagion, preferring a phone contact with a gynecologist or a midwife. $26.4 \%$ of our sample skipped some planned visit and $18.7 \%$ of them skipped planned exams or vaccines.

\section{The experience of delivering during the lockdown for new-mothers}

Table 3 describes the experience of delivery and of baby management during the lockdown. The overall response rate for these items was $>99 \%$. The mean gestational age at the time of delivery was $39.4 \pm 1.3$ weeks (range $36-42$ weeks). $84.2 \%$ of the partners had the possibility to assist during labor. $75.3 \%$ of women declared they were afraid of giving birth during the COVID-19 pandemic and they reported that the reality was as they expected in $50.8 \%$ of cases, better than expected in $36.2 \%$. 
Table 3

The experience of delivering during the lockdown for new-mothers

\begin{tabular}{|c|c|c|c|c|}
\hline & Parameter & Category & $\begin{array}{l}N= \\
139\end{array}$ & $\%$ \\
\hline \multirow{10}{*}{$\begin{array}{l}\text { Delivery and obstetrics } \\
\text { care }\end{array}$} & \multirow[t]{3}{*}{ Delivery mode } & Vaginal Eutocic & 96 & $69,1 \%$ \\
\hline & & Vaginal dystocic & 22 & $15,8 \%$ \\
\hline & & Cesarean section & 21 & $15,1 \%$ \\
\hline & \multirow[t]{2}{*}{ Presence of partner during delivery } & Yes & 117 & $84.2 \%$ \\
\hline & & No & 22 & $15.8 \%$ \\
\hline & \multirow[t]{2}{*}{ Afraid of receiving worst assistance because of the pandemic } & Yes & 60 & $44,1 \%$ \\
\hline & & No & 76 & $55,9 \%$ \\
\hline & \multirow[t]{3}{*}{ Reality versus expectations for you } & As expected & 70 & $50,7 \%$ \\
\hline & & Better & 50 & $36,2 \%$ \\
\hline & & Worst & 18 & $13,0 \%$ \\
\hline \multirow{22}{*}{$\begin{array}{l}\text { Neonatal care and } \\
\text { breastfeeding }\end{array}$} & \multirow[t]{2}{*}{ Required intensive neonatal care } & Yes & 15 & $10.8 \%$ \\
\hline & & No & 124 & $89.2 \%$ \\
\hline & \multirow[t]{2}{*}{ Afraid of receiving a worse neonatal assistance } & Yes & 36 & $26.0 \%$ \\
\hline & & No & 102 & $74.0 \%$ \\
\hline & \multirow[t]{3}{*}{ Reality versus expectations for your baby } & As expected & 83 & $59.7 \%$ \\
\hline & & Better & 42 & $30.2 \%$ \\
\hline & & Worst & 14 & $10.1 \%$ \\
\hline & \multirow[t]{3}{*}{ Influence of restrictions on neonatal management } & No influence & 43 & $30.9 \%$ \\
\hline & & $\begin{array}{l}\text { Negative } \\
\text { influence }\end{array}$ & 85 & $61.2 \%$ \\
\hline & & $\begin{array}{l}\text { Positive } \\
\text { influence }\end{array}$ & 11 & $7.9 \%$ \\
\hline & \multirow[t]{2}{*}{ Breastfeeding during hospital stay } & Yes & 131 & $94.0 \%$ \\
\hline & & No & 8 & $6.0 \%$ \\
\hline & \multirow[t]{3}{*}{ Type of nutrition } & $\begin{array}{l}\text { Exclusive } \\
\text { breastfeeding }\end{array}$ & 98 & $70.5 \%$ \\
\hline & & Formula feeding & 38 & $27.3 \%$ \\
\hline & & $\begin{array}{l}\text { Human donor } \\
\text { milk }\end{array}$ & 3 & $2.2 \%$ \\
\hline & \multirow[t]{2}{*}{ Continued breastfeeding after discharge } & Yes & 132 & $95.0 \%$ \\
\hline & & No & 7 & $5.0 \%$ \\
\hline & \multirow[t]{2}{*}{ Still breastfeeding at the time of survey } & Yes & 126 & $90.6 \%$ \\
\hline & & No & 13 & $9.4 \%$ \\
\hline & \multirow[t]{3}{*}{ Influence of restrictions on breastfeeding } & No influence & 78 & $56.1 \%$ \\
\hline & & $\begin{array}{l}\text { Negative } \\
\text { influence }\end{array}$ & 51 & $36.7 \%$ \\
\hline & & $\begin{array}{l}\text { Positive } \\
\text { influence }\end{array}$ & 10 & $7.2 \%$ \\
\hline
\end{tabular}




\begin{tabular}{|llll|}
\hline Parameter & Category & $\begin{array}{c}\mathbf{N}= \\
\mathbf{1 3 9}\end{array}$ \\
\hline $\begin{array}{lll}\text { Who supported you for breastfeeding after the discharge (more } \\
\text { than one choice available) }\end{array}$ & Midwife & 40 & $28.8 \%$ \\
\cline { 2 - 4 } & Partner & 27 & $19.4 \%$ \\
\cline { 2 - 4 } & Relative or friend & 18 & $12.9 \%$ \\
\cline { 2 - 4 } & Pediatrician & 4 & $2.9 \%$ \\
\cline { 2 - 4 } & No support & 74 & $53.2 \%$ \\
\hline
\end{tabular}

Overall, $61.1 \%$ of the new-mothers stated that the restrictive measures had a negative impact on their baby management, $28.1 \%$ declared they had no impact. Most of the respondents reported to have breastfed their babies during hospital staying (94.2\%), and about two-thirds of them started breastfeeding within the first two hours after delivery (64.7\%). During hospital staying, $70.5 \%$ of the babies were exclusively breastfed, $27.3 \%$ received formula feeding, and $2.2 \%$ received human donor milk. The majority of the newmothers declared to have continued to breastfed their babies when discharged at home (95.0\%), and most of them (91.7\%) stated they were still breastfeeding the babies at the time of the survey, reporting exclusive breastfeeding in $85.0 \%$ of cases and mixed with formula feeding in $5.8 \%$.

No impact of restrictive measures on breastfeeding was reported by $56.1 \%$ of the new-mothers, a negative impact by $36.7 \%$ and a positive one by $7.2 \%$. After hospital discharge, the respondents reported having received no support for breastfeeding in $53.2 \%$ of cases. In our sample, only 3 women (2.2\%) had a confirmed SARS-CoV-2 infection: all of them were separated from their newborns maintaining the possibility to feed them with expressed breast milk.

\section{Adjusted analysis for pregnant women}

Table 4 shows $\chi 2$ and prevalence across independent variables and three crucial outcomes in the pregnant women group: $\mathrm{PH} 4$ score from moderate to severe, difficulties in healthy eating and reduction in physical exercise. 
Table 4

chi 2 and Poission analysis for pregnant women main outcomes

\begin{tabular}{|c|c|c|c|c|c|c|c|c|c|c|c|c|c|}
\hline & & \multicolumn{4}{|c|}{ Anxiety and depression } & \multicolumn{4}{|c|}{ Difficulties in healthy eating } & \multicolumn{4}{|c|}{ Reduction in physical exercise } \\
\hline & & $\begin{array}{l}\mathrm{PH} 4 \\
\text { Mod } \\
\text { Seve }\end{array}$ & ate to & $\begin{array}{l}\text { Chi2 } \\
\mathrm{p} \\
\text { value }\end{array}$ & $\begin{array}{l}\text { PR (IC } \\
95 \%)\end{array}$ & Yes & & $\begin{array}{l}\text { Chi2 } \\
\mathrm{p} \\
\text { value }\end{array}$ & $\begin{array}{l}\text { PR (IC } \\
95 \%)\end{array}$ & Yes & & $\begin{array}{l}\text { Chi2 } \\
\mathrm{p} \\
\text { value }\end{array}$ & $\begin{array}{l}\text { PR (IC } \\
95 \%)\end{array}$ \\
\hline \multirow[t]{2}{*}{ Age } & $\begin{array}{l}\text { Less } \\
\text { than } 34\end{array}$ & 237 & $62,2 \%$ & & & 168 & $44,1 \%$ & & & 201 & $62,4 \%$ & & \\
\hline & From 35 & 141 & $63,5 \%$ & 0,75 & $\begin{array}{l}1.06 \\
(0.86- \\
1.31)\end{array}$ & 100 & $45,0 \%$ & 0,82 & $\begin{array}{l}1.09 \\
(0.84- \\
1.40)\end{array}$ & 112 & $60,9 \%$ & 0,73 & $\begin{array}{l}0.98 \\
(0.77- \\
1.23)\end{array}$ \\
\hline \multirow[t]{2}{*}{ Education } & $\begin{array}{l}\text { Less } \\
\text { than } \\
\text { Bachelor }\end{array}$ & 158 & $68,7 \%$ & & & 127 & $55,2 \%$ & & & 112 & $63,3 \%$ & & \\
\hline & $\begin{array}{l}\text { More } \\
\text { than } \\
\text { Bachelor }\end{array}$ & 219 & $58,9 \%$ & 0,02 & $\begin{array}{l}0.91 \\
(0.73- \\
1-13)\end{array}$ & 140 & $37,6 \%$ & $\dot{0}_{0,001}$ & $\begin{array}{l}0.7 \\
(0,54 \\
- \\
0.90)\end{array}$ & 200 & $61,0 \%$ & 0,61 & $\begin{array}{l}0.96 \\
(0.76- \\
1.21)\end{array}$ \\
\hline \multirow[t]{2}{*}{$\begin{array}{l}\text { Satisfaction } \\
\text { with } \\
\text { economical } \\
\text { resources }\end{array}$} & $\begin{array}{l}\text { Not } \\
\text { much } \\
\text { adeguate }\end{array}$ & 224 & $71,8 \%$ & & & 153 & $49,0 \%$ & & & 162 & $64,8 \%$ & & \\
\hline & $\begin{array}{l}\text { Very } \\
\text { adeguate }\end{array}$ & 151 & $52,4 \%$ & $<001$ & $\begin{array}{l}0.75 \\
(0.61- \\
0.94)\end{array}$ & 115 & $39,9 \%$ & 0,02 & $\begin{array}{l}0.92 \\
(0.71- \\
1.19)\end{array}$ & 150 & $59,3 \%$ & 0,20 & $\begin{array}{l}0.91 \\
(0.73- \\
1.14)\end{array}$ \\
\hline \multirow{2}{*}{$\begin{array}{l}\text { Satisfaction } \\
\text { with your } \\
\text { home }\end{array}$} & $\begin{array}{l}\text { Not } \\
\text { much }\end{array}$ & 172 & $73,8 \%$ & & & 119 & $51,1 \%$ & & & 129 & $65,5 \%$ & & \\
\hline & $\begin{array}{l}\text { Very } \\
\text { much }\end{array}$ & 206 & $55,7 \%$ & $<0,001$ & $\begin{array}{l}0,82 \\
(0.66- \\
1.01)\end{array}$ & 149 & $40,3 \%$ & 0,01 & $\begin{array}{l}0.85 \\
(0.66- \\
1.09)\end{array}$ & 184 & $59,5 \%$ & 0,18 & $\begin{array}{l}0.92 \\
(0.73- \\
1.17)\end{array}$ \\
\hline \multirow{2}{*}{$\begin{array}{l}\text { Partner } \\
\text { supporting }\end{array}$} & No & 78 & $77,2 \%$ & & & 60 & $59,4 \%$ & & & 52 & $66,7 \%$ & & \\
\hline & Yes & 300 & $59,8 \%$ & $<$ & $\begin{array}{l}0.81 \\
(0.63- \\
1.04)\end{array}$ & 208 & $41,4 \%$ & $<$ & $\begin{array}{l}0.72 \\
(0.54- \\
0.97)\end{array}$ & 261 & $61,0 \%$ & 0,34 & $\begin{array}{l}0.91 \\
(0.68- \\
1.23)\end{array}$ \\
\hline \multirow{2}{*}{$\begin{array}{l}\text { Contacts } \\
\text { With other } \\
\text { people }\end{array}$} & No & 240 & $63,3 \%$ & & & 156 & $41,2 \%$ & & & 199 & $62,2 \%$ & & \\
\hline & Yes & 138 & $61,6 \%$ & 0,67 & $\begin{array}{l}1.00 \\
(0.81- \\
1.24)\end{array}$ & 112 & $50,0 \%$ & 0,03 & $\begin{array}{l}1.24 \\
(0.97- \\
1.59)\end{array}$ & 114 & $61,3 \%$ & 0,84 & $\begin{array}{l}0.99 \\
(0.78- \\
1.25)\end{array}$ \\
\hline \multirow[t]{3}{*}{$\begin{array}{l}\text { Availability } \\
\text { of free time }\end{array}$} & $\begin{array}{l}\text { Less } \\
\text { than } \\
\text { before }\end{array}$ & 102 & $65,0 \%$ & & $\begin{array}{l}1.05 \\
(0.77- \\
1.43)\end{array}$ & 77 & $49,0 \%$ & & $\begin{array}{l}1.26 \\
(0.87- \\
1.84)\end{array}$ & 87 & $70,2 \%$ & & $\begin{array}{l}1.12 \\
(0.79- \\
1.57)\end{array}$ \\
\hline & $\begin{array}{l}\text { As } \\
\text { before }\end{array}$ & 71 & $65,7 \%$ & & & 45 & $41,7 \%$ & & & 55 & $63,2 \%$ & & \\
\hline & $\begin{array}{l}\text { More } \\
\text { than } \\
\text { before }\end{array}$ & 204 & $60,7 \%$ & 0,51 & $\begin{array}{l}1.01 \\
(0.77- \\
1.34)\end{array}$ & 145 & $43,2 \%$ & 0,38 & $\begin{array}{l}1.14 \\
(0.81- \\
1.60)\end{array}$ & 169 & $57,7 \%$ & 0,05 & $\begin{array}{l}0.92 \\
(0.67- \\
1.26)\end{array}$ \\
\hline \multirow{2}{*}{$\begin{array}{l}\text { Other } \\
\text { children at } \\
\text { home }\end{array}$} & No & 251 & $61,5 \%$ & & & 167 & $40,9 \%$ & & & 218 & $59,6 \%$ & & \\
\hline & Yes & 127 & $65,1 \%$ & 0,39 & $\begin{array}{l}1.00 \\
(0.80- \\
1.25)\end{array}$ & 101 & $51,8 \%$ & 0,01 & $\begin{array}{l}1.20 \\
(0.93- \\
1.55)\end{array}$ & 95 & $67,9 \%$ & 0,09 & $\begin{array}{l}1.16 \\
(0.90- \\
1.48)\end{array}$ \\
\hline \multirow[t]{2}{*}{ Trimester } & First & 38 & $69,1 \%$ & & & 16 & $29,1 \%$ & & & 39 & $90,7 \%$ & & \\
\hline & Second & 112 & $56,3 \%$ & & $\begin{array}{l}0.84 \\
(0.58- \\
1.22)\end{array}$ & 77 & $38,7 \%$ & & $\begin{array}{l}1.35 \\
(0.79- \\
2.32)\end{array}$ & 100 & $58,1 \%$ & & $\begin{array}{l}0.65 \\
(0.45- \\
0.94)\end{array}$ \\
\hline
\end{tabular}




\begin{tabular}{|c|c|c|c|c|c|c|c|c|c|c|c|c|}
\hline \multirow[b]{2}{*}{ Third } & \multicolumn{4}{|c|}{ Anxiety and depression } & \multicolumn{4}{|c|}{ Difficulties in healthy eating } & \multicolumn{4}{|c|}{ Reduction in physical exercise } \\
\hline & 224 & $64,9 \%$ & 0,08 & $\begin{array}{l}0.95 \\
(0.67- \\
1.35)\end{array}$ & 173 & $50,1 \%$ & $\hat{0}_{0,001}$ & $\begin{array}{l}1.69 \\
(1.01- \\
2.83)\end{array}$ & 172 & 59,99 & $\begin{array}{l}< \\
0,001\end{array}$ & $\begin{array}{l}0.67 \\
(0.47- \\
0.95)\end{array}$ \\
\hline
\end{tabular}

The prevalence of women with anxiety or depressive moderate to severe score was significantly higher in the group of women with a lower educational attainment and not satisfied with their economic resources and house. Additionally, it was also significantly lower in women who could count on the support of their partner. Finally, there is a trend showing a higher score in the first trimester.

While for most women restrictions gave them the chance to eat healthier, $20.2 \%$ of women reported difficulties in healthy eating. Likewise, a lower educational attainment, unsatisfaction with economic resources and house, and the lack of partner supporting are significantly associated to more difficulties in healthy eating during the lockdown. Furthermore, difficulties in healthy eating also directly correlated with having contacts with other people during the lockdown, having children at home and being in the third trimester of pregnancy.

Lastly, during the lockdown there was a significant reduction in physical activity, but this data is transversal to all the respondents and there are no significant differences between groups, except for two variables: women who had reduced the activity are those who during the lockdown had less free time than before, and who were in the first trimester.

\section{Discussion}

This study describes how pregnant women managed to cope with the lockdown in Italy. We found a high score for anxiety and depression, despite it cannot be compared to the same score on the same population before the pandemic. Our survey also suggests that the lockdown made it more difficult for pregnant women to exercise for 150 minutes per week in accordance with the ACOG guidelines (15), and we can assume that a reduction in physical exercise will affect the quality of life of pregnant women, as demonstrated in previous studies (16). On the other side it seems that staying at home facilitated the approach to healthy eating, for the group with the partner's support and a better socio-economic status. This is an interesting data that deserves more investigations and it is a starting point to develop new strategies for public health.

Among women who gave birth during the pandemic, although three-fourths of the respondents declared to be afraid of giving birth during such a complex period, the overall experience was as expected or better than expected for $87 \%$ of the population. Despite more than half of the new-mothers reported a negative influence on the baby's management and more than one-third of them reported a negative influence on their breastfeeding experience, the breastfeeding rate is consistent or even better than the ones before the pandemic (17), suggesting a slight discrepancy between expectations/perceptions and actual facts, probably due to the anxiety and depression characteristics found in our sample. More than half of new-mothers received no support for breastfeeding after hospital discharge; however almost all of the respondents have continued to breastfed their babies when discharged at home. It could be inferred that, in the impossibility to rely on external support, new-mothers have empowered their internal resources with satisfying results.

The high level of anxiety and depression is consistent with other studies (8) (9). The prevalence in the first trimester is confirmed (9) while, differently from another study (8) in our survey this data was not correlated with age, primiparity and area of living. The correlation with economic difficulties and education is consistent with the literature (16), and some studies suggest that COVID 19 pandemic may even worsen the social inequalities (18). Regarding the fact that a reduction of face to face visits could have occurred to women during the restrictions; a recent survey shows that patients are actually open to alternative models of prenatal care, including remote monitoring(19). Future survey could be done in the same population in order to find out if some changes are considered positively. According to a Cochrane review (20) communicating results of medical investigations by mobile phone messaging may make little or no difference to women's anxiety overall or in women with positive test results, but may reduce anxiety in women with negative test results. We cannot exclude that this method will be more largely implemented in future times, after the COVID-19 emergency and the lesson it gave us about face-to-face contact.

A higher prevalence of anxiety and depressive symptoms in pregnant and new-mother populations should be a public health issue, and screening for perinatal depression and anxiety should be considered during a pandemic. Under the circumstances of social distancing and isolation, psychological hotlines and online counseling would be a smart strategy to manage perinatal mental illness. The same

Page 12/16 
strategy would be useful to help new-mothers with the baby management. Healthcare professionals, should also ensure patients feel supported by continuing their routine prenatal care through tele-medicine visits (21). Clinicians might also consider recommending and encouraging "home" physical exercise, especially in women in the first trimester, who might be the most worried about the sudden change of their lives.

Isolation, increased stress, and sedentary lifestyle in pregnancy can also lead to adverse pregnancy outcomes, such as preterm birth, gestational diabetes and low birth weight (22)(23). This survey is also a baseline questionnaire for those women who gave consent to be contacted, and they will be followed up as a cohort in order to identify possible complications. In a further part of our project, we are going to describe in greater detail how the lockdown influenced neonatal outcomes.

The first limitation of the present study is related to the non-random sampling: women are enrolled by newspaper advertisements, social media and the snow-ball method; the completeness checks process was not exhaustive. A second limitation is the lack of validated questionnaire designed to capture such a delicate and unique moment. Third, the assessment of depressive and anxiety symptoms through a short scale which relied on a self-reported measure and does not provide a diagnosis. Although these limits, this is the first study to assess some aspects of the lifestyle of pregnant women and new-mothers during the lockdown in Italy. Besides, the web-based method is a strength because it gave us the opportunity to interview a geographically dislocated population during a short time in the lock-down period.

Given the unicity of this SAR-CoV2 pandemic we tried to give an overview of the experience of Italian pregnant women and new-mothers during the lockdown. Next steps will be to incorporate those findings in political choices. The WHO Executive Board recognizes the need

to include women in decision making for outbreak preparedness and response, however there is still inadequate women's representation in national and global COVID-19 policy spaces (24). It is also important that health professionals commit themselves to help pregnant women and new-mothers to overcome these difficult times.

\section{Declarations}

- Ethics approval and consent to participate:

the consent to participate was required and he participation in the survey was voluntary and anonymous. Approval was obtained from the local Ethical Committee (Comitato Etico Interaziendale Novara CE 71/20), which conformed to the principles embodied in the Declaration of Helsinki.

- Consent for publication: Not applicable

- Availability of data and materials:

The datasets generated and/or analysed during the current study are not publicly available due to privacy reasons but are available from the corresponding author on reasonable request.

- Competing interests:

The authors declare that they have no competing interests

- Funding: the research received no funding

- Authors' contributions:

Viviana STAMPINI, Conceptualization, Methodology, Software, Data Curation, writing-original draft

Alice MONZANI, Conceptualization, Data Curation, writing-original draft

Silvia CARISTIA, Formal analysis, Review \& Editing

Gianluigi FERRANTE, Review \& Editing, Visualization,

Martina GERBINO, Validation, Review \& Editing

Page 13/16 
Alberto DE PEDRINI, Project administration

Roberta AMADORI, Data Curation

Ivana RABBONE, Supervision

Daniela SURICO, Supervision

- Acknowledgements: The authors would like to acknowledge Mirela Kalcina, prof. M.A., for the proofreading.

- Authors' information (optional)

\section{References}

1. http://www.euro.who.int/en/health-topics/health-emergencies/coronavirus-covid-19/news/news/2020/3/who-announces-covid19-outbreak-a-pandemic.

2. Kraemer MUG, Yang C-H, Gutierrez B, Wu C-H, Klein B, Pigott DM, et al. The effect of human mobility and control measures on the COVID-19 epidemic in China. Science. 25 marzo 2020;eabb4218.

3. Rasmussen SA, Smulian JC, Lednicky JA, Wen TS, Jamieson DJ. Coronavirus Disease 2019 (COVID-19) and pregnancy: what obstetricians need to know. Am J Obstet Gynecol. maggio 2020;222(5):415-26.

4. Rasmussen SA, Jamieson DJ. Coronavirus Disease 2019 (COVID-19) and Pregnancy: Responding to a Rapidly Evolving Situation. Obstet Gynecol. maggio 2020;135(5):999-1002.

5. Wenham C, Smith J, Morgan R. COVID-19: the gendered impacts of the outbreak. The Lancet. marzo 2020;395(10227):846-8.

6. Della Gatta AN, Rizzo R, Pilu G, Simonazzi G. COVID19 during pregnancy: a systematic review of reported cases. Am J Obstet Gynecol. aprile 2020;S0002937820304385.

7. Du L, Gu YB, Cui MQ, et al. [Investigation on demands for antenatal care services among 2002 pregnant women during the epidemic of COVID-19 in Shanghai]. Zhonghua fu Chan ke za zhi. marzo 2020;55(3):160-165.

8. Wu Y, Zhang C, Liu H, Duan C, Li C, Fan J, et al. Perinatal depressive and anxiety symptoms of pregnant women along with COVID19 outbreak in China. Am J Obstet Gynecol. maggio 2020;S0002937820305342.

9. Saccone G, Florio A, Aiello F, Venturella R, De Angelis MC, Locci M, et al. Psychological Impact of COVID-19 in pregnant women. Am J Obstet Gynecol. maggio 2020;S0002937820305275.

10. Wang C, Pan R, Wan X, Tan Y, Xu L, Ho CS, et al. Immediate Psychological Responses and Associated Factors during the Initial Stage of the 2019 Coronavirus Disease (COVID-19) Epidemic among the General Population in China. Int J Environ Res Public Health. 6 marzo 2020;17(5):1729.

11. von Elm E, Altman DG, Egger M, Pocock SJ, Gøtzsche PC, Vandenbroucke JP. The Strengthening the Reporting of Observational Studies in Epidemiology (STROBE) Statement: Guidelines for reporting observational studies. Int J Surg. dicembre 2014;12(12):1495-9.

12. Eysenbach G. Improving the Quality of Web Surveys: The Checklist for Reporting Results of Internet E-Surveys (CHERRIES). J Med Internet Res. 29 settembre 2004;6(3):e34.

13. Marcano Belisario JS, Huckvale K, Saje A, Porcnik A, Morrison CP, Car J. Comparison of self administered survey questionnaire responses collected using mobile apps versus other methods. In: The Cochrane Collaboration, curatore. Cochrane Database of Systematic Reviews [Internet]. Chichester, UK: John Wiley \& Sons, Ltd; 2014 [citato 16 aprile 2020]. pag. MR000042. Available at: http://doi.wiley.com/10.1002/14651858.MR000042

14. Kroenke K, Spitzer RL, Williams JBW, Lowe B. An Ultra-Brief Screening Scale for Anxiety and Depression: The PHQ-4. Psychosomatics. 1 novembre 2009;50(6):613-21.

15. ACOG COMMITTEE OPINION Number 804 Meredith L. Birsner, MD; Cynthia Gyamfi-Bannerman, MD, MSc. Physical Activity and Exercise During Pregnancy and the Postpartum Period. 04/2020.

16. Lagadec N, Steinecker M, Kapassi A, Magnier AM, Chastang J, Robert S, et al. Factors influencing the quality of life of pregnant women: a systematic review. BMC Pregnancy Childbirth. dicembre 2018;18(1):455.

17. Davanzo R, Romagnoli C, Corsello G. Position Statement on Breastfeeding from the Italian Pediatric Societies. Ital J Pediatr. dicembre 2015;41(1):80. 
18. Onwuzurike C, Meadows AR, Nour NM. Examining Inequities Associated With Changes in Obstetric and Gynecologic Care Delivery During the Coronavirus Disease 2019 (COVID-19) Pandemic. Obstet Gynecol [Internet]. 30 aprile 2020 [citato 12 giugno 2020];Publish Ahead of Print. Available at: https://journals.Iww.com/10.1097/AOG.0000000000003933

19. Peahl AF, Novara A, Heisler M, Dalton VK, Moniz MH, Smith RD. Patient Preferences for Prenatal and Postpartum Care Delivery: A Survey of Postpartum Women. Obstet Gynecol. maggio 2020;135(5):1038-46.

20. Gurol-Urganci I, de Jongh T, Vodopivec-Jamsek V, Car J, Atun R. Mobile phone messaging for communicating results of medical investigations. Cochrane Consumers and Communication Group, curatore. Cochrane Database Syst Rev [Internet]. 13 giugno 2012 [citato 9 maggio 2020]; Available at: http://doi.wiley.com/10.1002/14651858.CD007456.pub2

21. Jago CA, Singh SS, Moretti F. Coronavirus Disease 2019 (COVID-19) and Pregnancy: Combating Isolation to Improve Outcomes. Obstet Gynecol [Internet]. 8 maggio 2020 [citato 12 giugno 2020];Publish Ahead of Print. Available at: https://journals.Iww.com/10.1097/AOG.0000000000003946

22. Elsenbruch S, Benson S, Rücke M, Rose M, Dudenhausen J, Pincus-Knackstedt MK, et al. Social support during pregnancy: effects on maternal depressive symptoms, smoking and pregnancy outcome. Hum Reprod. marzo 2007;22(3):869-77.

23. Shapiro GD, Fraser WD, Frasch MG, Séguin JR. Psychosocial stress in pregnancy and preterm birth: associations and mechanisms. J Perinat Med [Internet]. 1 gennaio 2013 [citato 12 giugno 2020];41(6). Available at: https://www.degruyter.com/view/j/jpme.2013.41.issue-6/jpm-2012-0295/jpm-2012-0295.xml

24. Mathieu Boniol, Michelle Mclsaac, Lihui Xu, Tana Wuliji, Khassoum Diallo, Jim Campbell. Gender equity in the health workforce: Analysis of 104 countries Health Workforce Working Paper 1.

\section{Figures}




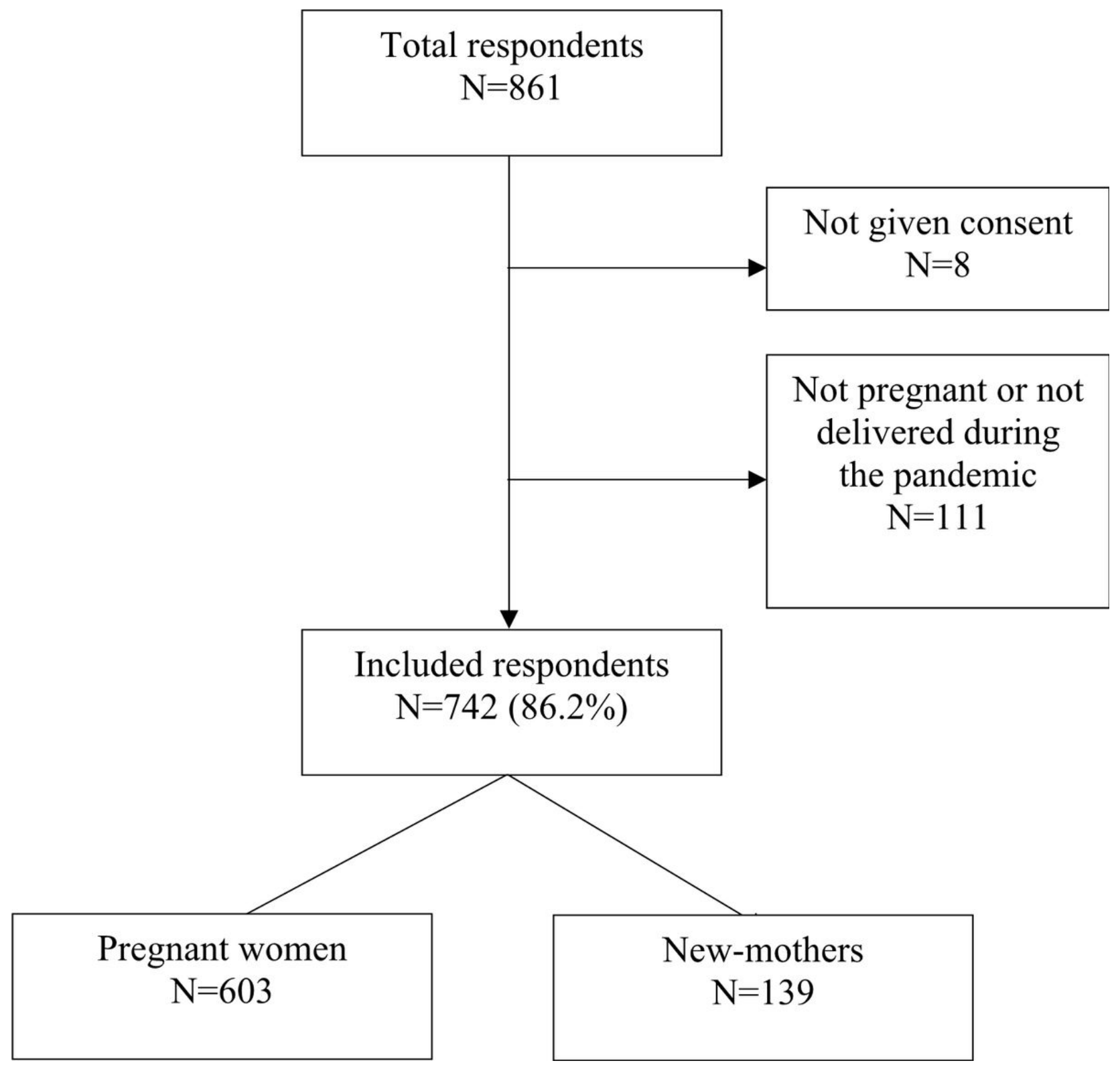

Figure 1

\section{Supplementary Files}

This is a list of supplementary files associated with this preprint. Click to download.

- Surveyinenglish.docx 